\title{
Substituen SMILE
}

$\mathrm{H}$

$\mathrm{O}=\mathrm{C}(\mathrm{N}[\mathrm{C} @ @ \mathrm{H}](\mathrm{C} 1=\mathrm{CC}=\mathrm{CC}=\mathrm{C} 1) \mathrm{CC}([\mathrm{O}-])=\mathrm{O}) \mathrm{CCCCC}(\mathrm{C}=\mathrm{C} 2)=[\mathrm{NH}+] \mathrm{C} 3=\mathrm{C} 2 \mathrm{CCCN} 3$

$\mathrm{F} \quad \mathrm{O}=\mathrm{C}(\mathrm{N}[\mathrm{C} @ @ \mathrm{H}](\mathrm{C} 1=\mathrm{CC}=\mathrm{CC}(\mathrm{F})=\mathrm{C} 1) \mathrm{CC}([\mathrm{O}-])=\mathrm{O}) \mathrm{CCCCC}(\mathrm{C}=\mathrm{C} 2)=[\mathrm{NH}+] \mathrm{C} 3=\mathrm{C} 2 \mathrm{CCCN} 3$

$\mathrm{CH} 3 \quad \mathrm{O}=\mathrm{C}(\mathrm{N}[\mathrm{C} @ @ \mathrm{H}](\mathrm{C} 1=\mathrm{CC}=\mathrm{CC}(\mathrm{C})=\mathrm{C} 1) \mathrm{CC}([\mathrm{O}-])=\mathrm{O}) \mathrm{CCCCC}(\mathrm{C}=\mathrm{C} 2)=[\mathrm{NH}+] \mathrm{C} 3=\mathrm{C} 2 \mathrm{CCCN} 3$

CF3 $\mathrm{O}=\mathrm{C}(\mathrm{N}[\mathrm{C} @ \mathrm{H}](\mathrm{C} 1=\mathrm{CC}=\mathrm{CC}(\mathrm{C}(\mathrm{F})(\mathrm{F}) \mathrm{F})=\mathrm{C} 1) \mathrm{CC}([\mathrm{O}-])=\mathrm{O}) \mathrm{CCCCC}(\mathrm{C}=\mathrm{C2})=[\mathrm{NH}+] \mathrm{C} 3=\mathrm{C} 2 \mathrm{CCCN} 3$

$\mathrm{OCH} 3 \quad \mathrm{O}=\mathrm{C}(\mathrm{N}[\mathrm{C} @ \mathrm{H}](\mathrm{C} 1=\mathrm{CC}=\mathrm{CC}(\mathrm{OC})=\mathrm{C} 1) \mathrm{CC}([\mathrm{O}-])=\mathrm{O}) \mathrm{CCCCC}(\mathrm{C}=\mathrm{C2})=[\mathrm{NH}+] \mathrm{C} 3=\mathrm{C} 2 \mathrm{CCCN} 3$

OCF3 $\mathrm{O}=\mathrm{C}(\mathrm{N}[\mathrm{C} @ \mathrm{H}](\mathrm{C} 1=\mathrm{CC}=\mathrm{CC}(\mathrm{OC}(\mathrm{F})(\mathrm{F}) \mathrm{F})=\mathrm{C} 1) \mathrm{CC}([\mathrm{O}-\mathrm{-})=\mathrm{O}) \mathrm{CCCCC}(\mathrm{C}=\mathrm{C} 2)=[\mathrm{NH}+] \mathrm{C} 3=\mathrm{C} 2 \mathrm{CCCN} 3$ 